\title{
Lozenge Tiling Function Ratios for Hexagons with Dents on Two Sides
}

\author{
Daniel Condon \\ Department of Mathematics \\ Indiana University \\ Bloomington Indiana, U.S.A. \\ dmcondon@indiana.edu
}

Submitted: Feb 12, 2020; Accepted: Aug 4, 2020; Published: Sep 18, 2020

(C) The author. Released under the CC BY license (International 4.0).

\begin{abstract}
We give a formula for the number of lozenge tilings of a hexagon on the triangular lattice with unit triangles removed from arbitrary positions along two non-adjacent, non-opposite sides. Our formula implies that for certain families of such regions, the ratios of their numbers of tilings are given by simple product formulas.
\end{abstract}

Mathematics Subject Classifications: $05 \mathrm{C} 70$

\section{Introduction}

The triangular lattice induces a tiling of the plane, by unit equilateral triangles, which contains no holes and where the triangles ${ }^{1}$ do not overlap. A region on the triangular lattice is a connected union of finitely many of those triangles. We say triangles that share an edge are adjacent. A lozenge on the triangular lattice is the union of two adjacent triangles, and a lozenge tiling or simply tiling of a region is a set of lozenges within that region, which cover the region, and which do not overlap. A region with at least one tiling is called tileable. A hexagonal region is said to be semiregular if its parallel sides are the same length. We identify congruent regions and let $H_{a, b, c}$ denote the semiregular hexagon(al region) with pairs of parallel sides of lengths $a, b$, and $c$.

A formula for the number of lozenge tilings of a semiregular hexagon was first given by MacMahon [23] in the context of plane partitions. A bijection between plane partitions and lozenge tilings was later given by David and Tomei [8]. Letting $\mathrm{M}(R)$ denote the number of lozenge tilings of a region $R$, MacMahon's formula can be expressed

$$
\mathrm{M}\left(H_{a, b, c}\right)=P(a, b, c), \quad \text { where } \quad P(a, b, c):=\prod_{i=1}^{c} \frac{(a+i)_{b}}{(i)_{b}}
$$

\footnotetext{
${ }^{1}$ When we say 'triangle' in this paper, we always mean a unit triangle on the lattice.
} 


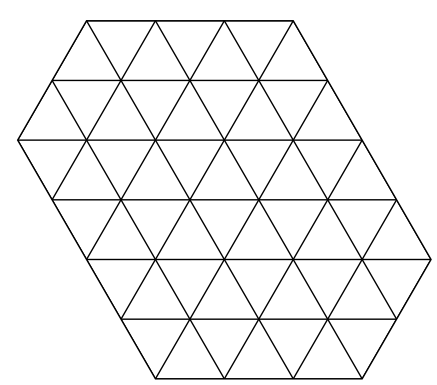

(a)

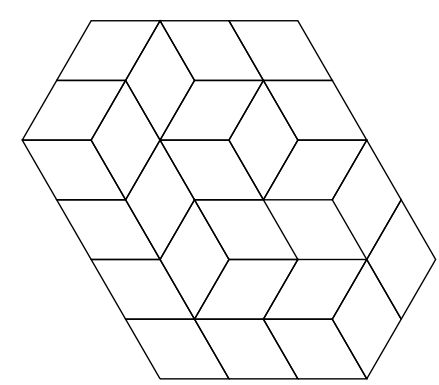

(b)

Figure 1: (a) $H_{3,4,2}$; (b) a tiling of $H_{3,4,2}$.

and $(x)_{y}$ is the Pochhammer symbol, $(x)_{y}=\prod_{i=0}^{y-1}(x+i)$; throughout this paper we consider the empty product to be 1 , so that $(x)_{0}=1$. When $R_{\bar{x}}$ denotes a family of regions defined by parameters $\bar{x}$ (as with $\left.\left\{H_{a, b, c}: a, b, c \in \mathbb{N}\right\}\right)^{2}$ we say that $\mathrm{M}\left(R_{\bar{x}}\right)$ denotes the tiling function of $R_{\bar{x}}$ with parameters $\bar{x}$. So $P(a, b, c)$ is the tiling function for semiregular hexagons.

Hexagons on the triangular lattice need not be semiregular, but the lengths of parallel sides necessarily differ by the same amount, say $t$. When $t>0$ we say the longer of each parallel pair is a long side and the rest are short sides. We can therefore define hexagonal regions by the lengths of their short sides $a, b, c$, and the difference $t$, using the notation $H_{a, b, c, t}$. The sides of the hexagon alternate around the perimeter between short and long, so without a loss of generality we will assume that the side lengths of the hexagon appear in the clockwise order $a, b+t, c, a+t, b, c+t$ starting from the northern side. The hexagon in Figure 2 (a) is $H_{4,3,2,4}$. Semiregular hexagons have $t=0$, so $H_{a, b, c}=H_{a, b, c, 0}$.

The region $H_{4,3,2,4}$ has no lozenge tilings at all. A lozenge covers exactly one uppointing triangle and one down-pointing triangle, so that a region can only be tileable if it contains the same number of triangles with each orientation: we call such a region balanced. The hexagon $H_{a, b, c, t}$ contains an excess of $t$ triangles with one orientation, so semiregular hexagons are the only hexagons which are balanced. The triangles in excess are those that lie along ${ }^{3}$ the long sides of $H_{a, b, c, t}$ (by our conventions, these are up-pointing).

In this paper we study regions we call dented hexagons, obtained by removing triangles from two long sides of a hexagon. We suppose $m$ triangles are removed from the northeast side, and $n$ triangles are removed from the northwest side. For balanced regions, this implies $t=n+m$. Figure 2 (b) gives one example of such a region. The black triangles have been removed from the region; we call these dents. The grey areas are part of the dented hexagon but are covered by forced lozenges, which are described at the end of

\footnotetext{
${ }^{2}$ We use $\mathbb{N}$ to denote the nonnegative integers.

${ }^{3}$ When we say a triangle lies along a side of the hexagon, we mean it shares an edge with the border of that side. When we say we a triangle is removed from a side, we mean that the removed triangle lies along that side.
} 


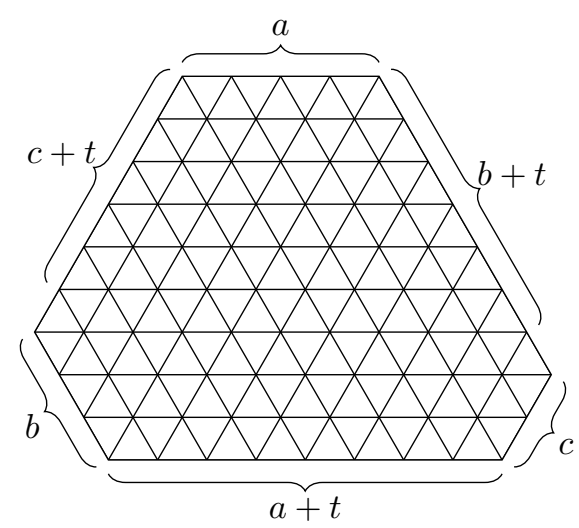

(a)

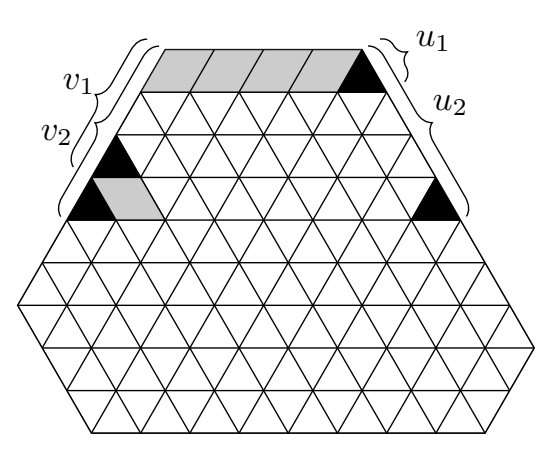

(b)

Figure 2: (a) $H_{4,3,2,4}$; (b) $H_{4,3,2,4,(1,4),(3,4)}$ with forced lozenges shaded grey. For this region $\underline{u_{1}}=5, \underline{u_{2}}=3$, and $\underline{v_{1}}=\underline{v_{2}}=2$.

\section{Section 2.}

Given non-negative integers $a, b, c, t$ suppose $\vec{u}=\left(u_{i}\right)_{i=1}^{m}$ and $\vec{v}=\left(v_{j}\right)_{j=1}^{n}$ are vectors of integers with $1 \leqslant u_{i}<u_{i+1} \leqslant b+t$ for $1 \leqslant i<m$, and $1 \leqslant v_{j}<v_{j+1} \leqslant c+t$ for $1 \leqslant j<n$, with $a>0$ or $u_{1}>1$ or $v_{1}>1{ }^{4}$ Then we use $H_{a, b, c, t, \vec{u}, \vec{v}}$ to denote the dented hexagon formed by removing dents from $H_{a, b, c, t}$ at locations indexed by $\vec{u}$ and $\vec{v}$. Specifically, we remove each $u_{i}$ th triangle from the northeast side of $H_{a, b, c, t}$ and each $v_{j}$ th triangle from the northwest side of $H_{a, b, c, t}$. The indexing in both cases starts at the northernmost triangle lying along each side. It is straightforward to check that $H_{a, b, c, t, \vec{u}, \vec{v}}$ with parameters as described is well-defined and unique.

Other natural parameters of dented hexagons we will use are $\underline{u_{i}}:=b+n+i-u_{i}$ and $v_{j}:=$ $c+m+j-v_{j}$. The parameter $u_{i}$ counts the number of triangles lying along the northeast side south of the dent indexed by $u_{i}$ which have not been removed from the region: these are the up-pointing triangles directly southeast of that dent, as shown in Figure 2 (b). The parameter $\underline{v_{j}}$ analogously counts up-pointing triangles directly southwest of the dent indexed by $v_{j}$.

The main result of this paper is a remarkably simple product formula for the ratio of the tiling functions of $H_{a, b, c, t, \vec{u}, \vec{v}}$ and $H_{0, b, c, t, \vec{u}, \vec{v}}$ so long as the latter region is well-defined. It follows that the first family of regions has a tiling function given by a simple product formula whenever the second family does, and we identify some instances of this. This is not the first paper to identify families of regions with simple ratios of tiling functions; similar results were recently found by Lai, Ciucu, Rohatgi, and Byun [1], [5], [6], [19],

\footnotetext{
${ }^{4}$ For the hexagon $H_{0, b, c, t}$, the northernmost triangle on the northwest side is the same as the northernmost triangle on the northeast side. For this reason, it is a little unclear what a dented hexagon with $a=0$ and $u_{1}=v_{1}=1$ should look like, since we cannot remove that triangle from the region twice. Since well-defined dented hexagons with $u_{1}=v_{1}=1$ have no tilings anyway (by Proposition 4 ) we do not dwell on this.
} 
[20], [21]. One specific subfamily of dented hexagons was studied by Lai, who found a lovely tiling function [18].

Eisenkölbl gave a tiling function for hexagonal regions with one dent on each long side (Theorem 1 from [9]); in the special case that one of these dents is at a corner of the region, tiling these is equivalent to tiling one of our dented hexagons of the form $H_{a, b, c, 2,\left(u_{1}\right),\left(v_{1}\right)}$. A general tiling function for hexagonal regions with any number of dents lying at arbitrary positions along their border was later discovered by Ciucu and Fischer (Theorem 3 from [2]) and presented as a generalization of Eisenkölbl's result, but that function is given only as the Pfaffian of a matrix. Gilmore in [13] gives tiling functions for even more general families of regions, in which the removed triangles need not lie along the boundary of the regions. Our main result gives a tiling function for dented hexagons and generalizes this special case of Eisenkölbl's result. In contrast the regions we discuss are themselves a special case of Ciucu and Fischer's, but our function's presentation highlights the effects of transforming the regions naturally by stretching.

\section{Further Background}

A region on the triangular lattice can be identified with a graph, so that each triangle corresponds to a vertex and adjacent triangles correspond to adjacent vertices - this is often called the planar dual of the region. This graph is bipartite, with its vertex bipartition defined by the orientations of the triangles, since triangles are only adjacent to triangles of the opposite orientation. A lozenge tiling of a region naturally partitions the triangles of that region into adjacent pairs, and so a lozenge tiling can be identified with a perfect matching on the planar dual of that region.

We will therefore borrow some graph-theoretic techniques. For an introduction to this perspective see [15], a classical paper by Kasteleyn which discusses connections between several types of problems in graph theory including matching problems ${ }^{5}$. Another classical paper on matching problems is [24] by Temperley and Fisher. These three authors were all pioneers of this field.

The following is a direct application of the Graph Splitting Lemma, which appears as Lemma 3.6 in a 2014 paper by Lai [17], and is implicit in earlier work by Ciucu [3].

Lemma 1 (Region Splitting Lemma). Let $R$ be a balanced region of the triangular lattice with a partition into regions $P$ and $Q$ such that triangles in $P$ which are adjacent to triangles in $Q$ are all of the same orientation, and that this orientation is not in excess within $P$. Then $\mathrm{M}(R)=\mathrm{M}(P) \cdot \mathrm{M}(Q)$, and in particular $\mathrm{M}(R)=0$ if either $P$ or $Q$ is not balanced.

We will also use Kuo's graphical condensation method. The following is a special case of his Theorem 5.4 from [16], expressed in the language of this paper.

\footnotetext{
${ }^{5}$ Perfect matchings in that paper are referred to as dimer coverings.
} 
Lemma 2. Let $R$ be a simply connected region of the triangular lattice, and let $\alpha, \beta, \gamma, \delta$ be triangles in $R$ of the same orientation which touch the boundary of $R$ at a corner or edge, so that the places they touch the boundary appear in the cyclic order $\alpha, \beta, \gamma, \delta$. Then

$$
\begin{aligned}
& \mathrm{M}(R-\alpha-\gamma) \cdot \mathrm{M}(R-\beta-\delta) \\
= & \mathrm{M}(R-\alpha-\delta) \cdot \mathrm{M}(R-\beta-\gamma)+\mathrm{M}(R-\alpha-\beta) \cdot \mathrm{M}(R-\gamma-\delta)
\end{aligned}
$$

It is a classical result of Lindström (Lemma 1 from [22]) that the signed weighted count $^{6}$ of families of non-intersecting paths from a set of sources $\left\{s_{i}: i \in[n]\right\}$ to a set of sinks $\left\{t_{j}: j \in[n]\right\}$ on a directed acyclic graph is given by the determinant of a matrix: namely an $n \times n$ matrix whose $(i, j)$ th entry is the weighted count of paths from $s_{i}$ to $t_{j}$. We henceforth refer to this as the path matrix. This actually generalizes an earlier independent result by Karlin and McGregor ((B) from [14]) regarding discrete Markov processes, which uses the determinant of an analogue to the path matrix to calculate the probability of $n$ particles starting and ending in specified states without two particles ever coinciding in a state. Gessel and Viennot later explored how Lindström's result applied to families of paths on the north-east-directed integer lattice, including identifying natural conditions for applying the result $[11,12]$. Their result developed into a very important method for counting tilings, especially when the determinant of the path matrix has a closed form. What follows is a restatement of their Corollary 2 from [12]:

Proposition 3. Let $S=\left\{\left(s_{i}, t_{i}\right): i \in[n]\right\}$ and $E=\left\{\left(p_{j}, q_{j}\right): j \in[n]\right\}$ be sets of coordinates on the square lattice $\mathbb{Z}^{2}$, such that every north-east lattice path from $\left(s_{i}, t_{i}\right)$ to $\left(p_{j}, q_{j}\right)$ intersects any north-east lattice path from $\left(s_{j}, t_{j}\right)$ to $\left(p_{i}, q_{i}\right)$ for $i \neq j$. The number of families of $n$ non-intersecting north-east lattice paths which each start at a point in $S$ and end at a point in $E$ is given by $\operatorname{det}\left(\left(a_{i, j}\right)_{i, j=1}^{n}\right)$, where $a_{i, j}=\left(\begin{array}{c}p_{j}+q_{j}-s_{i}-t_{i} \\ q_{j}-t_{i}\end{array}\right)$ is the number of north-east lattice paths from $\left(s_{i}, t_{i}\right)$ to $\left(p_{j}, q_{j}\right)$.

When a fixed lozenge occurs in every tiling of a region, we say that lozenge is forced. Often, a region contains a triangle which is adjacent to only one other triangle - the lozenge covering those two triangles is forced. One can remove the triangles covered by a forced lozenge to produce a new region, the tilings of which are in a natural bijection with the tilings of the original region; the bijection is defined by removing or replacing the forced lozenge. The new region may itself have a triangle only adjacent to one other triangle, which means it too is covered by a lozenge that is forced in the new region, and therefore in the original region. Removing triangles covered by forced lozenges yields a sequence of regions that all have the same number of tilings. Figure 2 (b) serves as an example. The forced lozenges are shown and colored grey.

\section{Main Results}

Our first remark about dented hexagons is a characterization of when they have any tilings at all.

\footnotetext{
${ }^{6}$ The signed weight of such a family is the product of the weights of each path, multiplied by the sign of the permutation $\sigma$ so that each path which starts at $s_{i}$ ends at $t_{\sigma(i)}$.
} 


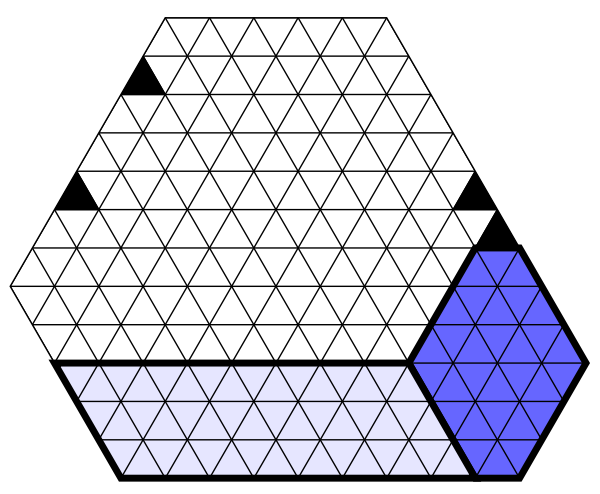

(a)

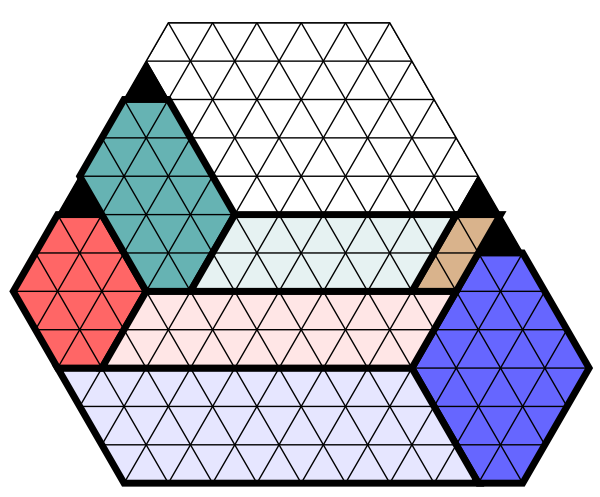

(b)

Figure 3: $H_{5,5,3,4,(5,6),(2,5)}$ depicted in (a) partitioned into three tileable regions; depicted in (b) partitioned into many regions.

Proposition 4. Let a balanced dented hexagon $H_{a, b, c, t, \vec{u}, \vec{v}}$ be given. Let $L_{N}$ be the Nth horizontal lattice line south of the northern side $e^{7}$ of $H_{a, b, c, t, \vec{u}, \vec{v}}$, and $\mu_{N}$ be the number of dents north of $L_{N}$. Then $H$ has a tiling iff $\mu_{N} \leqslant N$ for all $N \in \mathbb{N}$.

Proof. Suppose for some $N, \mu_{N}>N$; assume that $N$ is minimal such that this is the case. It is straightforward to check the region above $L_{N}$ is unbalanced. By Lemma 1, applied to the regions north and south of $L_{N}$, the dented hexagon has no tilings.

If $\mu_{N} \leqslant N$ for each $N$, we can exhibit a partition of the overall region into tileable regions, proving the existence of a tiling for the entire region. We induct on the number of dents.

If the region has no dents, it is a semiregular hexagon and is tileable. Suppose Proposition 4 holds for dented hexagons with fewer than $t$ dents. Suppose without a loss of generality that $u_{m} \geqslant v_{n}$; in other words the southernmost dent is on the northeast side of the region. We sketch the semiregular hexagon $H_{1, \underline{u_{m}, c}}$ directly underneath the southernmost dent, so that its northern side is the border of that dent. Figure 3 (a) depicts this hexagon (dark). We also sketch a parallelogram consisting of all triangles directly west of the southwest edge of the dark hexagon. This parallelogram is also depicted (light) in Figure 3 (a). Both the hexagon and parallelogram are tileable, and the rest of the region is tileable by the induction hypothesis; so the entire region is tileable.

The fact that $\mu_{t-1} \leqslant t-1$ implies that $u_{m} \geqslant t$, and $u_{m} \leqslant b$. This guarantees the northeast and southwest sides of the dark hexagon are of length $\leqslant b$, so that the light region west of the dark hexagon is actually a parallelogram. Had it been the case that $v_{n}>u_{m}$, we would reflect the picture across a vertical axis to get a depiction of $H_{a, c, b, t, \vec{v}, \vec{u}}$, find a partition of that region, and then reflect the picture again across the same axis to get a partition of $H_{a, b, c, t, \vec{u}, \vec{v}}$.

\footnotetext{
${ }^{7} L_{N}$ is then $\frac{\sqrt{3}}{2} N$ units south of the northern side of $H_{a, b, c, t, \vec{u}, \vec{v}}$.
} 
Our application of the inductive hypothesis in the proof implies that one could continue subdividing the entire region into parallelograms and semiregular hexagons. Such a subdivision is depicted in Figure 3 (b). Proposition 4 shows that untileable dented hexagons are characterized by having too many dents too far north, which yields the following consequence.

Corollary 5. Let $H=H_{a, b, c, t,\left(u_{i}\right)_{1}^{m},\left(v_{j}\right)_{1}^{n}}$ and $H^{\prime}=H_{a^{\prime}, b^{\prime}, c^{\prime}, t,\left(u_{i}^{\prime}\right)_{1}^{m},\left(v_{j}^{\prime}\right)_{1}^{n}}$ be dented hexagons with $u_{i} \leqslant u_{i}^{\prime}$ and $v_{j} \leqslant v_{j}^{\prime}$ for each $i, j$. If $H$ is tileable then so is $H^{\prime}$.

Our main result is that families of dented hexagons with fixed parameters, $b, c, t, \vec{u}, \vec{v}$ have a tiling function given by the following rational function in $a$ :

Theorem 6. Where $H_{a, b, c, t,\left(u_{i}\right)_{1}^{m},\left(v_{j}\right)_{1}^{n}}$ is a tileable dented hexagon,

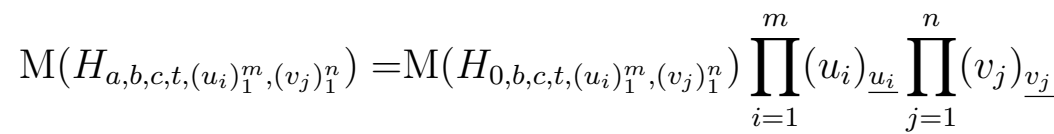

$$
\begin{aligned}
& \times \frac{P(a, b+n, c+m)}{\prod_{i=1}^{m}\left(a+u_{i}\right)_{\underline{u_{i}}} \prod_{j=1}^{n}\left(a+v_{j}\right)_{\underline{v_{j}}}} .
\end{aligned}
$$

In fact, the right hand side of this expression is a polynomial in $a$ as a result of Lemma 7 which we prove in the next section. When $H_{0, b, c, t, \vec{u}, \vec{v}}$ has a simple tiling function, Theorem 6 shows that $H_{a, b, c, t, \vec{u}, \vec{v}}$ also has a simple tiling function. In particular, this explains a result found by Lai (Theorem 3.1 from [18], with $q=1$ ), that when the dents lying along each side of the region are adjacent and $v_{n}=u_{m}$ the tiling function for the region is a simple product formula. We discuss this and related results in Section 6.

\section{Groundwork}

In this section we lay the technical foundation for the arguments that follow.

Lemma 7. If $H_{0, b, c, t, \vec{u}, \vec{v}}$ is a tileable dented hexagon, then $\mathrm{M}\left(H_{a, b, c, t, \vec{u}, \vec{v}}\right)$ is a polynomial in a when fixing $b, c, t$ and the vectors $\vec{u}$ and $\vec{v}$.

Proof. The tilings of $H_{a, b, c, t,\left(u_{i}\right)_{1}^{m},\left(v_{j}\right)_{1}^{n}}$ are in bijection with families of $(b+n)$ non-intersecting lattice paths which start at points along the southwest boundaries of the dented hexagon and end at points along the northeast boundaries. This bijection is explained briefly in Figure 4. Using this type of bijection is a standard technique when enumerating lozenge tilings, and similar examples of the technique explained in greater detail can be found in Sections 4 and 5 of [4].

The start points along the southwest side of the region can be interpreted as having coordinates $\{(-i, i): i \in[b]\}$, and the start points from the dents would then have coordinates $\left\{\left(-b, b+c+t+1-v_{i}\right): i \in[n]\right\}$. The end points then have coordinates $\left\{(a-b-t-1+j, b+c+t+1-j): j \in[b+t]-\left\{u_{i}: i \in[m]\right\}\right\}$. It is clear these satisfy the path-intersection condition of Proposition 3 when each set is labeled with indices increasing from north to south. 


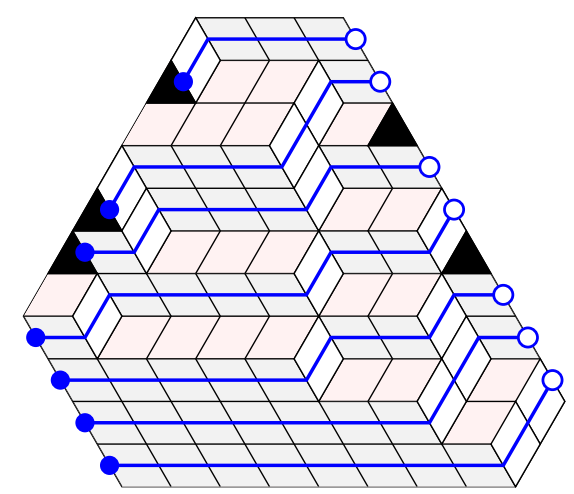

(a)

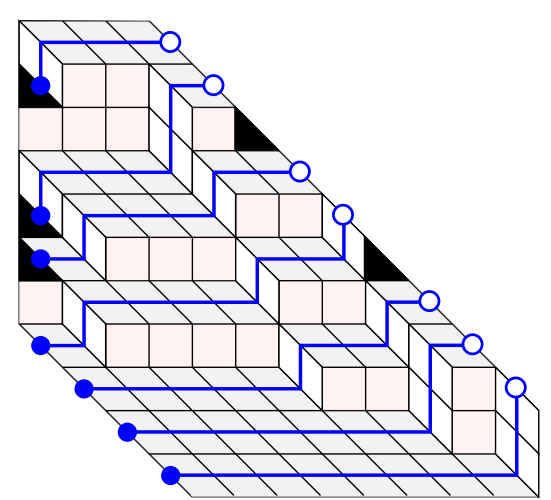

(b)

Figure 4: Tilings of $H_{3,4,2,5,(3,6),(2,5,6)}$ correspond to lattice paths which start at points on edges along the southwest borders of the region (including along the southwest side of the hexagon and also along the western dents) and end at points on edges along the northeast borders, the steps of which are directly east or northeast. The correspondence is as follows: given a tiling, place dots in the middle of these edges as exhibited in (a). Then, connect each dot by a path to the middle of the opposite edge of the lozenge containing it, and then to the middle of the opposite edge of the next lozenge, and so on until the dots are connected by paths. Warping the picture as in (b) transforms the paths into non-intersecting north-east lattice paths. This process is reversible.

Applying Proposition 3, the number of lattice paths with these start and end points is given by the determinant of a matrix, the entries of which are of the form $\left(\begin{array}{c}a-t-1+v_{i} \\ v_{i}-j\end{array}\right)$ and $\left(\begin{array}{c}a+c \\ b+c+t+1-j-i\end{array}\right)$. Since each entry in the matrix is a polynomial in $a$, so is the determinant.

The arguments that follow are largely about the proportionality of functions of $a$. For $p, q$ rational functions in $a$ (and possibly other indeterminates) we will use the nonstandard notation

$$
p(a) \stackrel{a}{=} q(a)
$$

to mean there exists some (not identically zero) rational function $k$ which is independent of $a$ and $k \cdot p(a)=q(a)$. It is easy to check $\stackrel{a}{=}$ is an equivalence relation and satisfies the following properties, where $p_{1}, p_{2}$, and $q$ are rational functions in $a$, and neither $\left(p_{1}+p_{2}\right)$ nor $q$ is identically zero.

$$
\begin{aligned}
& p_{1}(a) \stackrel{a}{=} p_{2}(a) \Rightarrow p_{1}(a)+p_{2}(a) \stackrel{a}{=} p_{1}(a) \stackrel{a}{=} p_{2}(a) \\
& p_{1}(a) \stackrel{a}{=} p_{2}(a) \Longleftrightarrow p_{1}(a) q(a) \stackrel{a}{=} p_{2}(a) q(a)
\end{aligned}
$$

We will make use of the following technical lemma. 
Lemma 8. For $d$ an integer, y a positive integer, and $z$ a nonnegative integer,

$$
P(a+d, y-1, z+1) \stackrel{a}{=} P(a+d, y, z) \frac{(a+d+z+1)_{y-1}}{(a+d+y)_{z}} .
$$

Proof. It is straightforward to show from the definition of $P(x, y, z)$ that $\frac{P(x, y, z+1)}{P(x, y, z)}=$ $\frac{(x+z+1)_{y}}{(z+1)_{y}}$; in turn it is straightforward to show

$$
P(x, y-1, z+1)=P(x, y, z) \frac{(y)_{z}(x+z+1)_{y-1}}{(x+y)_{z}(z+1)_{y-1}} .
$$

Relation (5) follows from equation (6) where $x=a+d$.

\section{$5 \quad$ Proof of Theorem 6}

For $b, c \in \mathbb{N}$ and $\left(u_{i}\right)_{1}^{m},\left(v_{j}\right)_{1}^{n}$ vectors of positive integers so that there exists a well-defined

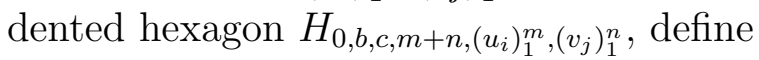

$$
f_{b, c,\left(u_{i}\right)_{1}^{m},\left(v_{j}\right)_{1}^{n}}(a):=\frac{P(a, b+n, c+m)}{\prod_{i=1}^{m}\left(a+u_{i}\right)_{\underline{u_{i}}} \prod_{j=1}^{n}\left(a+v_{j}\right)_{\underline{v_{j}}}} .
$$

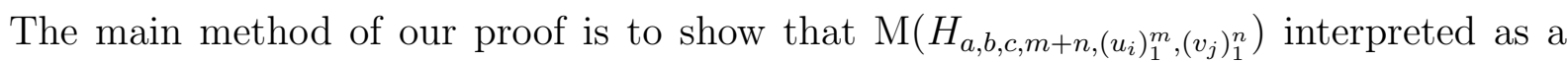
function of $a$ is proportional to $f$. We will do this by showing $f$ is a polynomial in $a$ which can be defined recursively and obeys the same recursion as the tiling function. Observe that for tileable regions the equation in Theorem 6 can be expressed

$$
\mathrm{M}\left(H_{a, b, c, t, \vec{u}, \vec{v}}\right) \stackrel{a}{=} f_{b, c, \vec{u}, \vec{v}}(a) .
$$

It will sometimes be convenient for us to assume that $u_{1}>1$ or $v_{1}>1$. A dented hexagon with $u_{1}=v_{1}=1$ has no tilings by Proposition 4 , in which case Theorem 6 holds trivially. In the case that $u_{1}=1$, and $v_{1}>1$ or $n=0$, the top row of triangles of the region $H_{a, b, c, t, \vec{u}, \vec{v}}$ are covered by forced lozenges, and may be removed from the region. Figure 5 (a) depicts an example of this. Removing the forced lozenges gives the region $H_{a+1, b, c, t-1,\left(u_{i}-1\right)_{i=2}^{m},\left(v_{j}-1\right)_{j=1}^{n}}$, which thus has the same tiling function as $H_{a, b, c, t, \vec{u}, \vec{v}}$. We will show that our definition of $f_{b, c, \vec{u}, \vec{v}}$ respects this and the analogous case with $v_{1}=1$.

If $\underline{u_{m}}=0$ (or $\underline{v}_{n}=0$ ), then the southeast (or southwest) side of the region is covered by forced lozenges. If $u_{m}=0$, then $H_{a, b, c, t, \vec{u}, \vec{v}}$ thus has the same tiling function as $H_{a, b, c+1, t-1,\left(u_{i}\right)_{i=1}^{m-1}, \vec{v}}$, which is the region that is obtained by removing the forced lozenges. This is depicted in Figure 5 (b). We will show our definition of $f_{b, c, \vec{u}, \vec{v}}$ respects this fact, and the analogous fact when $\underline{v_{n}}=0$.

Lemma 9. If $H_{a, b, c, t,\left(u_{i}\right)_{1}^{m},\left(v_{j}\right)_{1}^{n}}$ is tileable then

a. If $u_{1}=1$, and $v_{1}>1$ or $n=0$, then $f_{b, c, \vec{u}, \vec{v}}(a) \stackrel{a}{=} f_{b, c,\left(u_{i}-1\right)_{2}^{m},\left(v_{j}-1\right)_{1}^{n}}(a+1)$. 


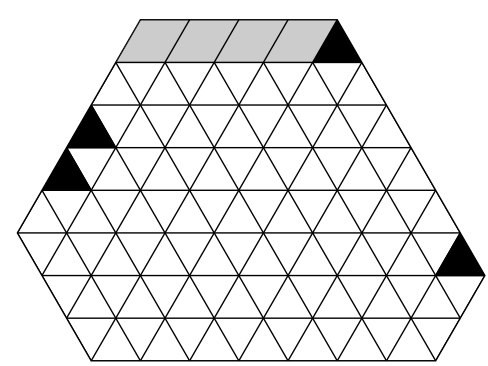

(a)

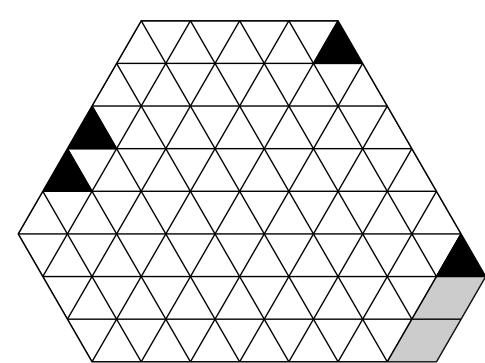

(b)

Figure 5: $H_{4,3,2,3,(1,6),(3,4)}$ with forced lozenges shown in grey; (a) the top row is forced since $u_{1}=1$; (b) the southeast side is forced since $u_{m}=0$.

b. If $v_{1}=1$, and $u_{1}>1$ or $m=0$, then $f_{b, c, \vec{u}, \vec{v}}(a) \stackrel{a}{=} f_{b, c,\left(u_{i}-1\right)_{1}^{m},\left(v_{j}-1\right)_{2}^{n}}(a+1)$.

c. If $\underline{u_{m}}=0$ then $f_{b, c, \vec{u}, \vec{v}}(a)=f_{b, c+1,\left(u_{i}\right)_{i=1}^{m-1},\left(v_{j}\right)_{1}^{n}}(a)$.

d. If $\underline{v_{n}}=0$ then $f_{b, c, \vec{u}, \vec{v}}(a)=f_{b+1, c,\left(u_{i}\right)_{i=1}^{m},\left(v_{j}\right)_{1}^{n-1}(a) .}$

Proof. These identities are straightforward to check when written explicitly.

Let $\vec{\varnothing}$ denote the empty vector. We will now verify that Theorem 6 holds when $\vec{u}=\vec{\varnothing}$. The case where $\vec{v}=\vec{\varnothing}$ follows by symmetry.

Lemma 10. The dented hexagon $H_{a, b, c, n, \vec{\varnothing},\left(v_{j}\right)_{1}^{n}}$ is tileable and $\mathrm{M}\left(H_{a, b, c, n, \vec{\varnothing},\left(v_{j}\right)_{1}^{n}}\right) \stackrel{a}{=} f_{b, c, \vec{\varnothing}, \vec{v}}(a)$ where

$$
f_{b, c, \vec{\varnothing}, \vec{v}}(a):=\frac{P(a, b+n, c)}{\prod_{j=1}^{n}\left(a+v_{j}\right)_{\underline{v_{j}}}} .
$$

This is equivalent to a result by Cohn, Larsen, and Propp which gives a formula for the number of tilings of a trapezoid with dents lying along its long base (see Section 2 of [7]), but the equivalence is not obvious so we will prove this result directly using Kuo Condensation.

Proof. Dented hexagons with dents on just one side are tileable by Proposition 4.

We will induct on $n+c$, with base cases at $n=0$ and $c=0$. Note that if $c=0$ then the region has a unique tiling 8 and $f_{b, 0, \vec{\gamma}, \vec{v}}(a):=\frac{P(a, b+n, 0)}{\prod_{j=1}^{n}\left(a+v_{j}\right)_{0}}=1$. In the case $n=0$, equation (7) reduces to MacMahon's formula (1).

For our inductive hypothesis, assume (7) holds for dented hexagons $H_{a, b^{\prime}, c^{\prime}, n^{\prime}, \vec{\varnothing},\left(v_{j}^{\prime}\right)_{1}^{n^{\prime}}}$ with $c^{\prime}+n^{\prime}<c+n$. Consider dented hexagons $H_{a, b, c, n, \vec{\varnothing},\left(v_{j}\right)_{1}^{n}}$.

\footnotetext{
${ }^{8}$ The entire northwest side is comprised of dents, and the unique tiling extends to the unique tiling of the $a \times(b+n)$ parallelogram.
} 


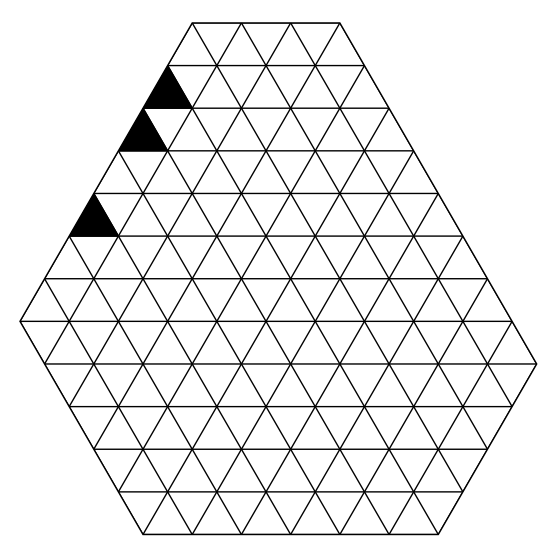

(a)

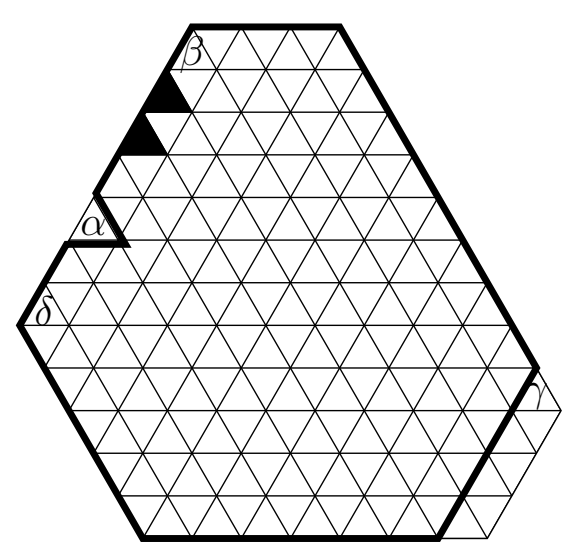

(b)

Figure 6: (a) $H_{3,5,4,3, \vec{\varnothing},(2,3,5)}$; (b) $R_{3}$ with $\alpha, \beta, \gamma, \delta$ labeled, and the border of $H_{3,5,4,3, \vec{\varnothing},(2,3,5)}$ depicted with a thick line.

In the case $v_{1}=1, H_{a, b, c, n, \vec{\varnothing},\left(v_{j}\right)_{1}^{n}}$ has forced lozenges along its northern side so has the same tiling function as $H_{a+1, b, c, n-1, \vec{\varnothing},\left(v_{j}-1\right)_{2}^{n}}$, so

$$
\begin{aligned}
\mathrm{M}\left(H_{a, b, c, n, \vec{\varnothing},\left(v_{j}\right)_{1}^{n}}\right) & \stackrel{a}{=} \mathrm{M}\left(H_{a+1, b, c, n-1, \vec{\varnothing},\left(v_{j}-1\right)_{2}^{n}}\right) \\
(\text { by IH }) & \stackrel{a}{=} f_{b, c, \vec{\varnothing},\left(v_{j}-1\right)_{2}^{n}}(a+1) \\
\text { (by Lemma 9b) } & \stackrel{a}{=} f_{b, c, \vec{\varnothing},\left(v_{j}\right)_{1}^{n}}(a) .
\end{aligned}
$$

In the case $\underline{v_{n}}=0, H_{a, b, c, n, \vec{\varnothing},\left(v_{j}\right)_{1}^{n}}$ has forced lozenges along its southwest side so has the same tiling function as $H_{a, b+1, c, n-1, \vec{\varnothing},\left(v_{j}\right)_{1}^{n-1}}$, so

$$
\begin{aligned}
& \mathrm{M}\left(H_{a, b, c, n, \vec{\varnothing},\left(v_{j}\right)_{1}^{n}}\right) \stackrel{a}{=} \mathrm{M}\left(H_{a, b+1, c, n-1, \vec{\varnothing},\left(v_{j}\right)_{1}^{n-1}}\right) \\
& \text { (by IH) } \stackrel{a}{=} f_{b, c+1, \vec{\varnothing},\left(v_{j}\right)_{1}^{n-1}}(a) \\
& \text { (by Lemma 9d) } \stackrel{a}{=} f_{b, c, \vec{\varnothing},\left(v_{j}\right)_{1}^{n}}(a) \text {. }
\end{aligned}
$$

We therefore assume that $n>0, v_{1}>1$, and $\underline{v}_{n}>0$. Regard $H_{a, b, c, n, \vec{\varnothing}, \vec{v}}$ as a subregion of the (unbalanced) region $R_{a}=H_{a, b, c-1, n+1, \vec{\varnothing},\left(v_{j}+1\right)_{j=1}^{n-1}}$. Within each region $R_{a}$, let $\alpha$ be the triangle indexed by $v_{n}$, let $\beta$ be the northernmost triangle lying along the northwest side, let $\gamma$ be the southernmost triangle lying along the northeast side, and let $\delta$ be the southernmost triangle lying along the northwest side. These placements are depicted in Figure 6.

We will apply Kuo Condensation (Lemma 2) to $R_{a}, \alpha, \beta, \gamma, \delta$. Figure 7 depicts each region referenced in the condensation formula, in some cases with forced lozenges shaded (we regard these as not being part of the region). Note they are all balanced dented hexagons. Since these regions can be regarded as dented hexagons with dents on only 

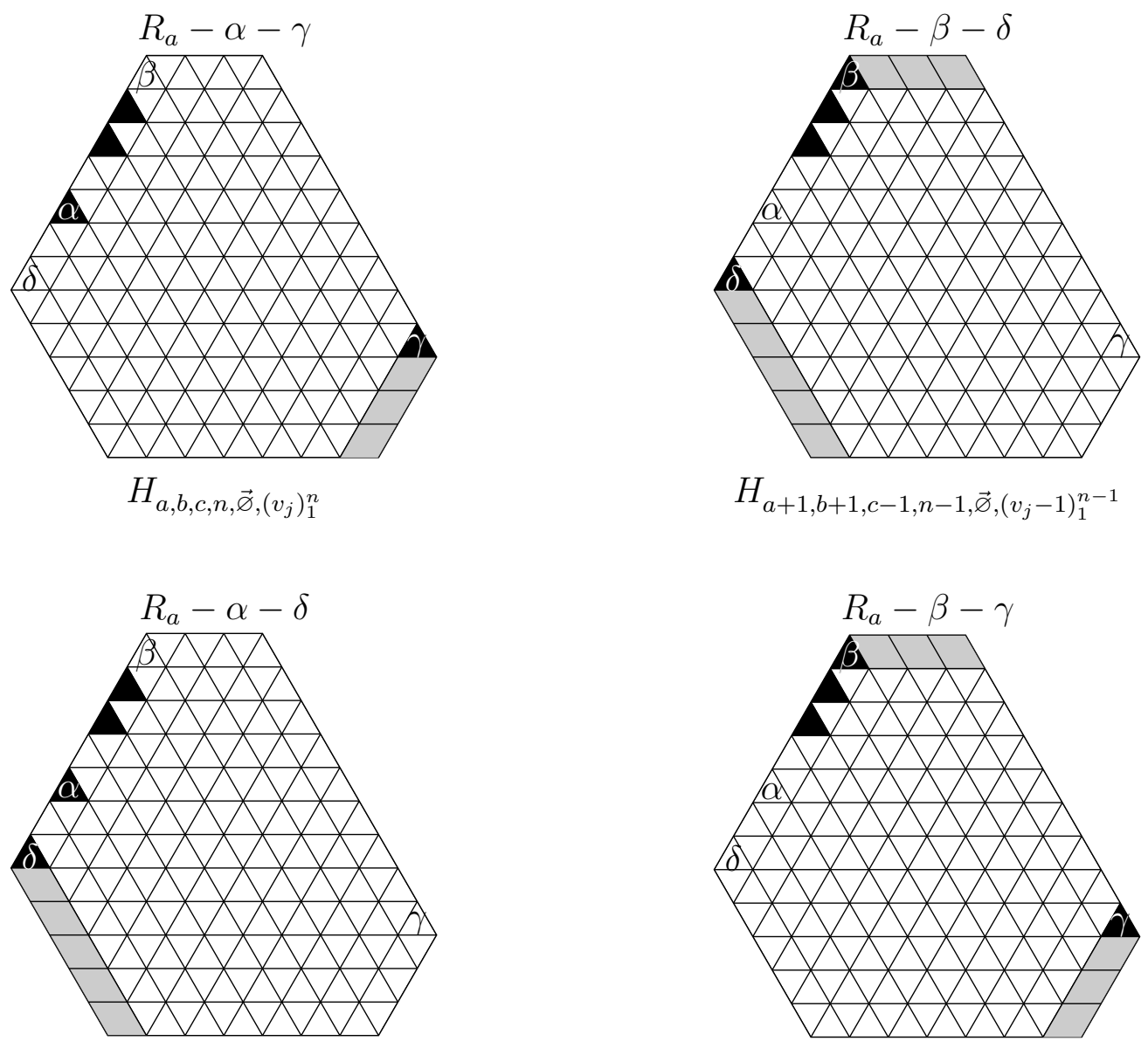

$H_{a, b+1, c-1, n, \vec{\varnothing},\left(v_{j}\right)_{1}^{n}}$

$H_{a+1, b, c, n-1, \vec{\varnothing},\left(v_{j}-1\right)_{1}^{n-1}}$
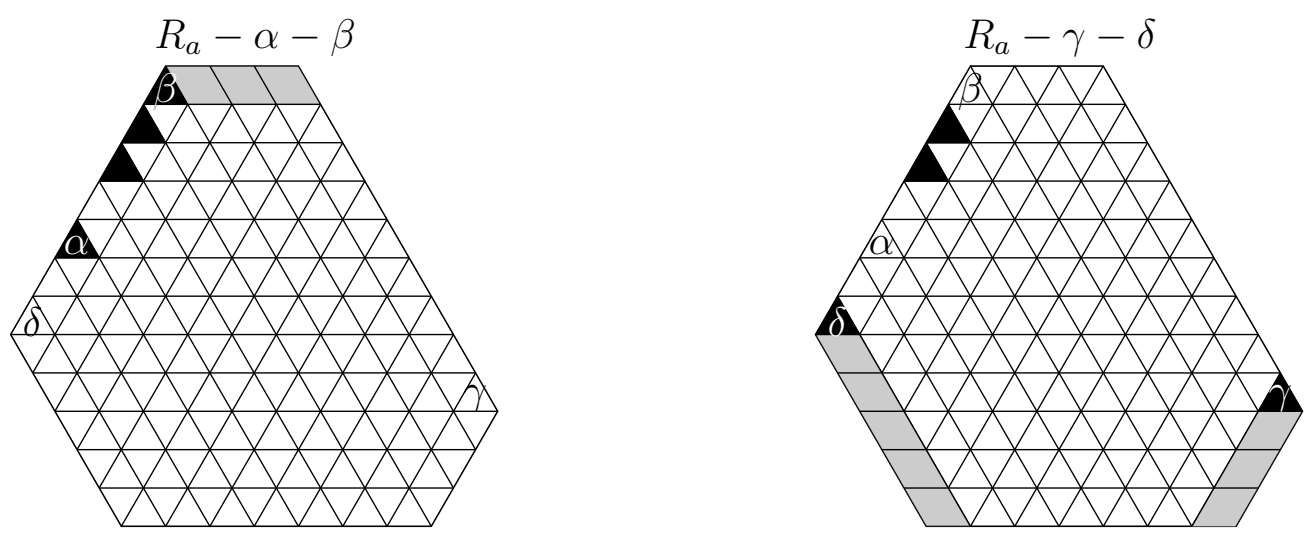

$H_{a+1, b, c-1, n, \vec{\varnothing},\left(v_{j}-1\right)_{1}^{n}}$

$H_{a, b+1, c, n-1, \vec{\varnothing},\left(v_{j}\right)_{1}^{n-1}}$

Figure 7: $R_{a}$ with two of $\alpha, \beta, \gamma, \delta$ removed and forced lozenges shaded. 
one side, each is tileable by Proposition 4 . The parameter $c+n$ is strictly minimal on $R_{a}-\alpha-\gamma$, so we may apply the inductive hypothesis to each of the other regions.

Recall Lemma 2 states

$M(R-\alpha-\gamma) M(R-\beta-\delta)=M(R-\alpha-\delta) M(R-\beta-\gamma)+M(R-\alpha-\beta) M(R-\gamma-\delta)$.

Removing forced lozenges as depicted in Figure 7, we can rewrite this:

$$
\begin{aligned}
& M\left(H_{a, b, c, n, \vec{\varnothing},\left(v_{j}\right)_{1}^{n}}\right) M\left(H_{a+1, b+1, c-1, n-1, \vec{\varnothing},\left(v_{j}-1\right)_{1}^{n-1}}\right) \\
& =M\left(H_{a, b+1, c-1, n, \vec{\varnothing},\left(v_{j}\right)_{1}^{n}}\right) M\left(H_{a+1, b, c, n-1, \vec{\varnothing},\left(v_{j}-1\right)_{1}^{n-1}}\right) \\
& +M\left(H_{a+1, b, c-1, n, \vec{\varnothing},\left(v_{j}-1\right)_{1}^{n}}\right) M\left(H_{a, b+1, c, n-1, \vec{\varnothing},\left(v_{j}\right)_{1}^{n-1}}\right) .
\end{aligned}
$$

Applying the inductive hypothesis to each region except $H_{a, b, c, n, \vec{\varnothing},\left(v_{j}\right)_{1}^{n}}$, this implies

$$
\begin{aligned}
& M\left(H_{a, b, c, n, \vec{\varnothing},\left(v_{j}\right)_{1}^{n}}\right) \cdot \frac{P(a+1, b+n, c-1)}{\prod_{j=1}^{n-1}\left(a+v_{j}\right)_{\underline{v_{j}}}} \\
& \stackrel{a}{=} \frac{P(a, b+n+1, c-1)}{\prod_{j=1}^{n}\left(a+v_{j}\right)_{\underline{v_{j}}-1}} \cdot \frac{P(a+1, b+n-1, c)}{\prod_{j=1}^{n-1}\left(a+v_{j}\right)_{v_{j}+1}} \\
& +\frac{P(a+1, b+n, c-1)}{\prod_{j=1}^{n}\left(a+v_{j}\right)_{\underline{v_{j}}}} \cdot \frac{P(a, b+n, c)}{\prod_{j=1}^{n-1}\left(a+v_{j}\right)_{\underline{v_{j}}}} .
\end{aligned}
$$

We shall show that when the term $M\left(H_{a, b, c, n, \vec{\varnothing},\left(v_{j}\right)_{1}^{n}}\right)$ is replaced with $f_{b, c, \vec{\varnothing},\left(v_{j}\right)_{1}^{n}}(a)$, the products on each line of (9) are $\stackrel{a}{=}$-equivalent, so that

$$
M\left(H_{a, b, c, n, \vec{\varnothing},\left(v_{j}\right)_{1}^{n}}\right) \stackrel{a}{=} f_{b, c, \vec{\varnothing},\left(v_{j}\right)_{1}^{n}}(a)
$$

by relations (3) and (4). Making this replacement, it is easy to see that

$$
f_{b, c, \vec{\varnothing},\left(v_{j}\right)_{1}^{n}}(a) \cdot \frac{P(a+1, b+n, c-1)}{\prod_{j=1}^{n-1}\left(a+v_{j}\right)_{\underline{v_{j}}}}=\frac{P(a+1, b+n, c-1)}{\prod_{j=1}^{n}\left(a+v_{j}\right)_{\underline{v_{j}}}} \cdot \frac{P(a, b+n, c)}{\prod_{j=1}^{n-1}\left(a+v_{j}\right)_{\underline{v_{j}}}}
$$

so it remains to show that the two products on the last two lines of (9) are $\stackrel{a}{=}$-equivalent. We rewrite

$$
\begin{aligned}
& \prod_{j=1}^{n}\left(a+v_{j}\right)_{\underline{v_{j}-1}}=\prod_{j=1}^{n}\left(a+v_{j}\right)_{\underline{v_{j}}} /(a+c)_{n} \\
& \prod_{j=1}^{n-1}\left(a+v_{j}\right)_{\underline{v_{j}+1}}=(a+c+1)_{n-1} \prod_{j=1}^{n-1}\left(a+v_{j}\right)_{v_{j}} .
\end{aligned}
$$

We can therefore rewrite the product on the second line of $(9)$ :

$$
\frac{P(a, b+n+1, c-1)}{\prod_{j=1}^{n}\left(a+v_{j}\right)_{\underline{v_{j}}-1}} \cdot \frac{P(a+1, b+n-1, c)}{\prod_{j=1}^{n-1}\left(a+v_{j}\right)_{\underline{v_{j}}}+1}
$$


(by eqns. $(10),(11))=\frac{P(a, b+n+1, c-1)}{\prod_{j=1}^{n}\left(a+v_{j}\right)_{\underline{v_{j}}}} \cdot \frac{P(a+1, b+n-1, c)}{\prod_{j=1}^{n-1}\left(a+v_{j}\right)_{\underline{v_{j}}}} \cdot \frac{(a+c)_{n}}{(a+c+1)_{n-1}}$

$$
\begin{aligned}
\text { (by Lemma 8) } \stackrel{\text { a }}{=} \frac{P(a, b+n, c)}{\prod_{j=1}^{n}\left(a+v_{j}\right)_{\underline{v_{j}}}} \cdot \frac{(a+b+n+1)_{c-1}}{(a+c)_{b+n}} \\
\\
\times \frac{P(a+1, b+n, c-1)}{\prod_{j=1}^{n-1}\left(a+v_{j}\right)_{\underline{v_{j}}}} \cdot \frac{(a+1+c)_{b+n-1}}{(a+b+n+1)_{c-1}} \cdot \frac{(a+c)_{n}}{(a+c+1)_{n-1}} \\
=\frac{P(a+1, b+n, c-1)}{\prod_{j=1}^{n}\left(a+v_{j}\right)_{\underline{v_{j}}}} \cdot \frac{P(a, b+n, c)}{\prod_{j=1}^{n-1}\left(a+v_{j}\right)_{\underline{v_{j}}}},
\end{aligned}
$$

which is exactly the product on the third line of (9). This completes the proof.

Note that $H_{0, b, c, n, \vec{\varnothing},\left(v_{j}\right)_{1}^{n}}$ has forced lozenges at its northern tip that when removed leave

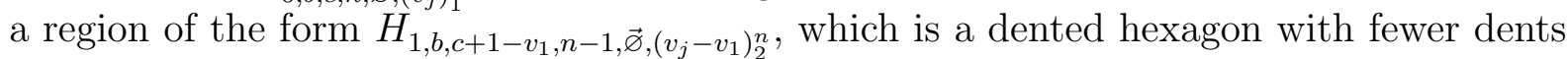
which still all lie along the northwest side. Through repeated application of Lemma 10, one can obtain a complete product formula for $\mathrm{M}\left(H_{a, b, c, n, \vec{\varnothing}, \vec{v}}\right)$; in particular, when $a=b=0$, this may be regarded as an independent proof for the number of tilings of a trapezoid with dents lying along its long base. This result is first mentioned in the literature by Cohn, Larsen, and Propp (see Section 2 of [7]) who rephrased an earlier result by Gelfand and Tsetlin [10] regarding monotone triangles. Our main theorem can be interpreted as a generalization of that result.

We are now ready to prove Theorem 6 . The proof is similar to that of Lemma 10, and uses that lemma as a base case.

Proof of Theorem 6 . We shall show by induction that if $H_{a, b, c, t, \vec{u}, \vec{v}}$ is tileable then

$$
\mathrm{M}\left(H_{a, b, c, t, \vec{u}, \vec{v}}\right) \stackrel{a}{=} f_{b, c, \vec{u}, \vec{v}}(a)
$$

where

$$
f_{b, c, \vec{u}, \vec{v}}(a):=\frac{P(a, b+n, c+m)}{\prod_{i=1}^{m}\left(a+u_{i}\right)_{\underline{u_{i}}} \prod_{j=1}^{n}\left(a+v_{j}\right)_{\underline{v_{j}}}} .
$$

We will induct on the number of dents $m+n$, using $m=0$ and $n=0$ as base cases. In these cases, relation (12) follows immediately from Lemma 10.

For our inductive hypothesis, suppose relation (12) holds for dented hexagons with fewer than $m+n$ dents. Consider a tileable dented hexagon with dents indexed by $\left(u_{i}\right)_{1}^{m}$, and $\left(v_{j}\right)_{1}^{n}$.

In the case where $\underline{u_{m}}=0, H_{a, b, c, t,\left(u_{i}\right)_{1}^{m},\left(v_{j}\right)_{1}^{n}}$ has forced lozenges along its southeast side and therefore has the same tiling function as $H_{a, b, c+1, t-1,\left(u_{i}\right)_{1}^{m-1},\left(v_{j}\right)_{1}^{n}}$, so

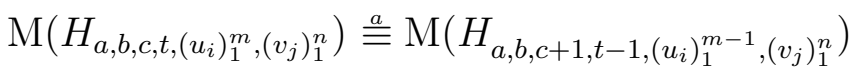

$$
\begin{aligned}
& (\mathrm{IH}) \stackrel{a}{=} f_{b, c+1,\left(u_{i}\right)_{1}^{m-1},\left(v_{j}\right)_{1}^{n}}(a)
\end{aligned}
$$




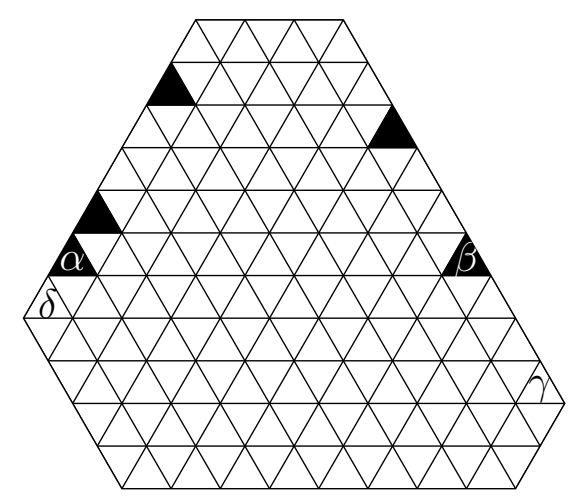

(a)

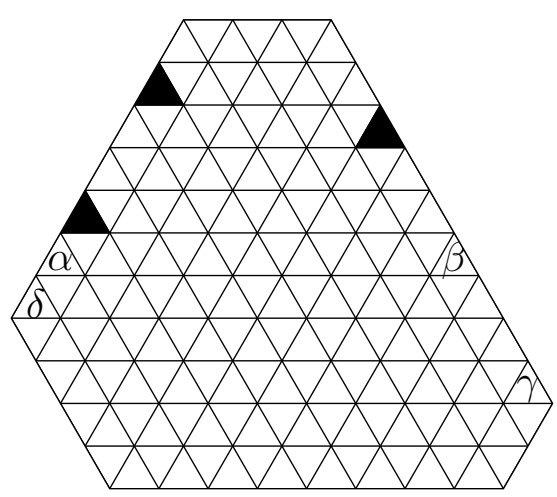

(b)

Figure 8: (a) $H_{3,4,2,5,(3,6),(2,5,6)}$; (b) $R_{3}$ with $\alpha, \beta, \gamma, \delta$ labeled.

$$
\text { (Lemma 9c) } \stackrel{a}{=} f_{b, c,\left(u_{i}\right)_{1}^{m},\left(v_{j}\right)_{1}^{n}}(a) \text {. }
$$

Relation (12) holds when $\underline{v}_{n}=0$ by a similar argument.

Assume therefore that $m, n>0, \underline{u_{m}}>0$ and $\underline{v_{n}}>0$. Regard $H_{a, b, c, t,\left(u_{i}\right)_{1}^{m},\left(v_{j}\right)_{1}^{n}}$ as a

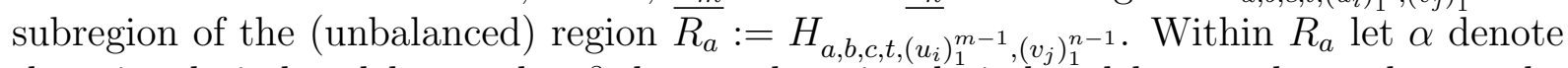
the triangle indexed by $v_{n}$, let $\beta$ denote the triangle indexed by $u_{m}$, let $\gamma$ denote the southernmost triangle lying along the northeast side of $R_{a}$, and let $\delta$ denote the southernmost triangle lying along the northwest side of $R_{a}$. Since $m, n>0$ and $u_{m}>0$ and $\underline{v}_{n}>0$ these locations are clearly defined within $R_{a}$ and are distinct, as depicted in Figure 8.

We will apply Kuo Condensation (Lemma 2) to $R_{a}, \alpha, \beta, \gamma, \delta$. Figure 9 depicts each region referenced in the condensation formula, in some cases with forced lozenges shaded (we regard these as not being part of the region). Note they are all families of tileable dented hexagons.

Since $H_{a, b, c, t, \vec{u}, \vec{v}}$ is tileable so are each of these regions by Corollary 5 . The number of dents is strictly minimal on $R_{a}-\alpha-\beta$, so we may apply the inductive hypothesis to each of the other regions. Lemma 2 can be expressed

$$
M(R-\alpha-\beta) M(R-\gamma-\delta)=M(R-\alpha-\gamma) M(R-\beta-\delta)-M(R-\alpha-\delta) M(R-\beta-\gamma)
$$

Removing forced lozenges as depicted in Figure 9, we can rewrite this:

$$
\begin{aligned}
& M\left(H_{\left.a, b, c, t,\left(u_{i}\right)_{1}^{m},\left(v_{j}\right)_{1}^{n}\right)}\right) M\left(H_{a, b+1, c+1, t-2,\left(u_{i}\right)_{1}^{m-1},\left(v_{j}\right)_{1}^{n-1}}\right)= \\
& M\left(H_{a, b, c+1, t-1,\left(u_{i}\right)_{1}^{m-1},\left(v_{j}\right)_{1}^{n}}\right) M\left(H_{a, b+1, c, t-1,\left(u_{i}\right)_{1}^{m},\left(v_{j}\right)_{1}^{n-1}}\right) \\
&-M\left(H_{a, b+1, c, t-1,\left(u_{i}\right)_{1}^{m-1},\left(v_{j}\right)_{1}^{n}}\right) M\left(H_{a, b, c+1, t-1,\left(u_{i}\right)_{1}^{m},\left(v_{j}\right)_{1}^{n-1}}\right) .
\end{aligned}
$$




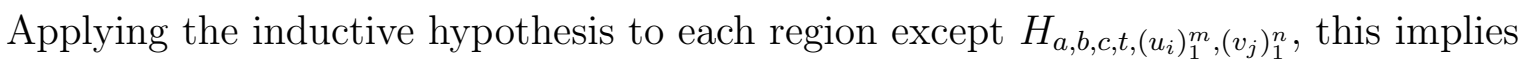

$$
\begin{aligned}
& M\left(H_{a, b, c, t,\left(u_{i}\right)_{1}^{m},\left(v_{j}\right)_{1}^{n}}\right) \cdot \frac{P(a, b+n, c+m)}{\prod_{i=1}^{m-1}\left(a+u_{i}\right)_{\underline{u_{i}}} \prod_{j=1}^{n-1}\left(a+v_{j}\right)_{\underline{v_{j}}}} \\
& \stackrel{a}{=} \frac{P(a, b+n, c+m)}{\prod_{i=1}^{m-1}\left(a+u_{i}\right)_{\underline{u_{i}}} \prod_{j=1}^{n}\left(a+v_{j}\right)_{\underline{v}_{j}}} \cdot \frac{P(a, b+n, c+m)}{\prod_{i=1}^{m}\left(a+u_{i}\right)_{\underline{u_{i}}} \prod_{j=1}^{n-1}\left(a+v_{j}\right)_{\underline{v_{j}}}} \\
& -\frac{P(a, b+n+1, c+m-1)}{\prod_{i=1}^{m-1}\left(a+u_{i}\right)_{\underline{u_{i}}+1} \prod_{j=1}^{n}\left(a+v_{j}\right)_{\underline{v_{j}}-1}} \cdot \frac{P(a, b+n-1, c+m+1)}{\prod_{i=1}^{m}\left(a+u_{i}\right)_{\underline{u_{i}}-1} \prod_{j=1}^{n-1}\left(a+v_{j}\right)_{\underline{v_{j}}+1}} .
\end{aligned}
$$

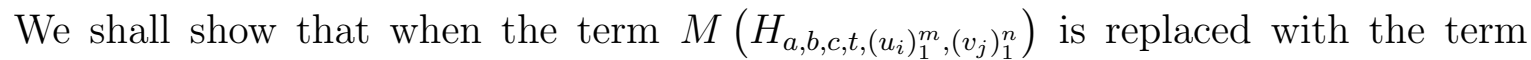
$f_{b, c,\left(u_{i}\right)_{1}^{m},\left(v_{j}\right)_{1}^{n}}(a)$, the products on each line of $(14)$ are $\stackrel{a}{=}$-equivalent, so that

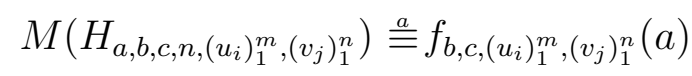

by relations (3) and (4). It is easy to see that

$$
\begin{aligned}
f_{b, c,\left(u_{i}\right)_{1}^{m},\left(v_{j}\right)_{1}^{n}}(a) \cdot & \frac{P(a, b+n, c+m)}{\prod_{i=1}^{m-1}\left(a+u_{i}\right)_{\underline{u_{i}}} \prod_{j=1}^{n-1}\left(a+v_{j}\right)_{v_{j}}} \\
& =\frac{P(a, b+n, c+m)}{\prod_{i=1}^{m-1}\left(a+u_{i}\right)_{\underline{u_{i}}} \prod_{j=1}^{n}\left(a+v_{j}\right)_{\underline{v_{j}}}} \frac{P(a, b+n, c+m)}{\prod_{i=1}^{m}\left(a+u_{i}\right)_{\underline{u_{i}}} \prod_{j=1}^{n-1}\left(a+v_{j}\right)_{v_{j}}}
\end{aligned}
$$

so it remains to show that the two products on the last two lines of (14) are $\stackrel{a}{=}$-equivalent. We will manipulate the terms on the third line to show this, employing the following analogues to equations (10) and (11):

$$
\begin{aligned}
& \prod_{j=1}^{k}\left(a+v_{j}\right)_{{\underline{v_{j}}}_{-1}}=\prod_{j=1}^{k}\left(a+v_{j}\right)_{\underline{v_{j}}} /(a+c+m)_{k} \\
& \prod_{i=1}^{k}\left(a+u_{i}\right)_{\underline{u_{i}-1}}=\prod_{i=1}^{k}\left(a+u_{j}\right)_{\underline{u_{i}}} /(a+b+n)_{k} \\
& \prod_{j=1}^{k}\left(a+v_{j}\right)_{\underline{v_{j}}+1}=(a+c+m+1)_{k} \prod_{j=1}^{k}\left(a+v_{j}\right)_{\underline{v_{j}}} \\
& \prod_{i=1}^{k}\left(a+u_{i}\right)_{\underline{u_{i}-1}}=(a+b+n+1)_{k} \prod_{i=1}^{k}\left(a+u_{j}\right)_{\underline{u_{i}}} .
\end{aligned}
$$

We can therefore rewrite:

$$
\frac{P(a, b+n+1, c+m-1)}{\prod_{i=1}^{m-1}\left(a+u_{i}\right)_{\underline{u_{i}}+1} \prod_{j=1}^{n}\left(a+v_{j}\right)_{\underline{v_{j}}-1}} \cdot \frac{P(a, b+n-1, c+m+1)}{\prod_{i=1}^{m}\left(a+u_{i}\right)_{\underline{u_{i}}-1} \prod_{j=1}^{n-1}\left(a+v_{j}\right)_{\underline{v_{j}}}+1}
$$

(eqns. (16)-(19) $)=\frac{P(a, b+n+1, c+m-1)}{\prod_{i=1}^{m-1}\left(a+u_{i}\right)_{\underline{u_{i}}} \prod_{j=1}^{n}\left(a+v_{j}\right)_{\underline{v_{j}}}} \cdot \frac{(a+c+m)_{n}}{(a+b+n+1)_{m-1}}$ 

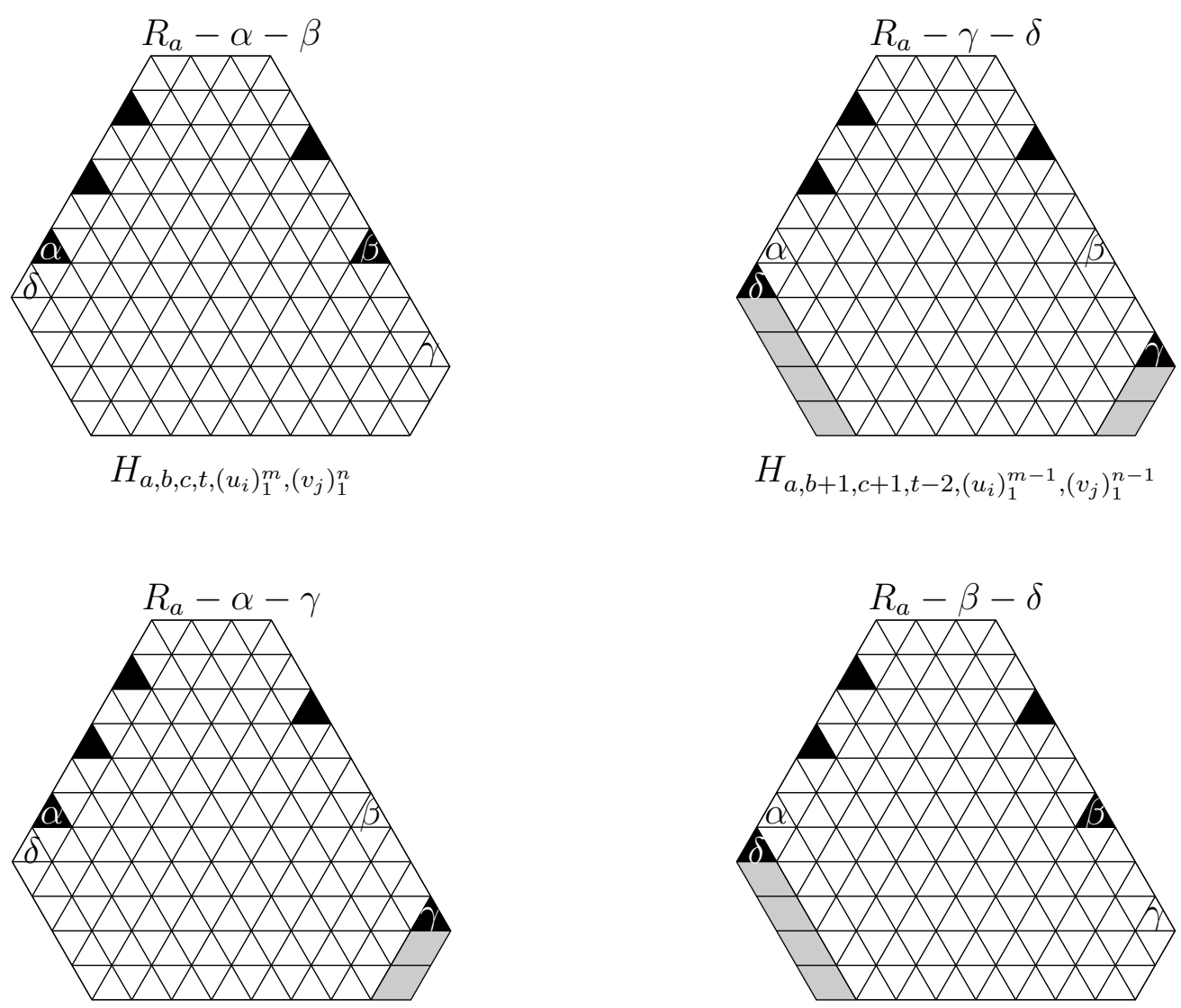

$H_{a, b, c+1, t-1,\left(u_{i}\right)_{1}^{m-1},\left(v_{j}\right)_{1}^{n}}$

$$
H_{a, b+1, c, t-1,\left(u_{i}\right)_{1}^{m},\left(v_{j}\right)_{1}^{n-1}}
$$
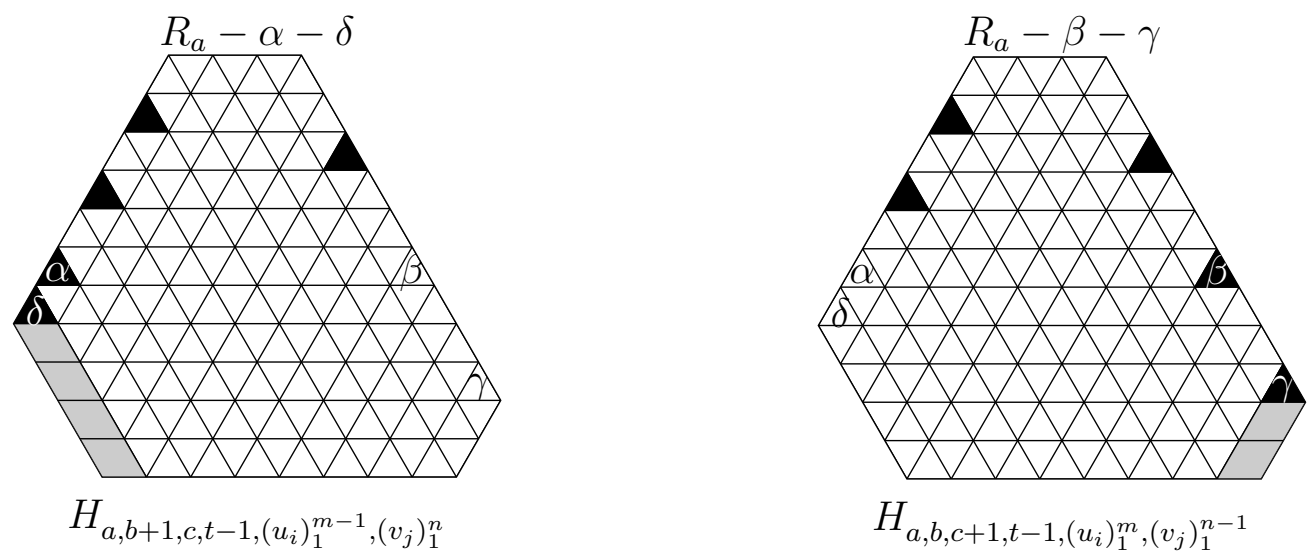

Figure 9: $R_{a}$ with two of $\alpha, \beta, \gamma, \delta$ removed and forced lozenges shaded. 


$$
\begin{aligned}
& \times \frac{P(a, b+n-1, c+m+1)}{\prod_{i=1}^{m}\left(a+u_{i}\right)_{\underline{u_{i}}} \prod_{j=1}^{n-1}\left(a+v_{j}\right)_{\underline{v_{j}}}} \cdot \frac{(a+b+n)_{m}}{(a+c+m+1)_{n-1}} \\
& \text { (by Lemma } 8) \stackrel{a}{=} \frac{P(a, b+n, c+m)}{\prod_{i=1}^{m-1}\left(a+u_{i}\right)_{\underline{u_{i}}} \prod_{j=1}^{n}\left(a+v_{j}\right)_{\underline{v_{j}}}} \cdot \frac{(a+b+n+1)_{c+m-1}}{(a+c+m)_{b+n}} \cdot \frac{(a+c+m)_{n}}{(a+b+n+1)_{m-1}} \\
& \times \frac{P(a, b+n, c+m)}{\prod_{i=1}^{m}\left(a+u_{i}\right)_{\underline{u_{i}}} \prod_{j=1}^{n-1}\left(a+v_{j}\right)_{\underline{v_{j}}}} \cdot \frac{(a+c+m+1)_{b+n-1}}{(a+b+n)_{c+m}} \cdot \frac{(a+b+n)_{m}}{(a+c+m+1)_{n-1}} \\
& =\frac{P(a, b+n, c+m)}{\prod_{i=1}^{m-1}\left(a+u_{i}\right)_{\underline{u_{i}}} \prod_{j=1}^{n}\left(a+v_{j}\right)_{\underline{v_{j}}}} \cdot \frac{P(a, b+n, c+m)}{\prod_{i=1}^{m}\left(a+u_{i}\right)_{\underline{u_{i}}} \prod_{j=1}^{n-1}\left(a+v_{j}\right)_{\underline{v_{j}}}},
\end{aligned}
$$

which is exactly the product from third line from (14). This is the last case in the inductive step, so relation (12) holds in general.

So given $H_{a, b, c, t,\left(u_{i}\right)_{1}^{m},\left(v_{j}\right)_{1}^{n}}$ a tileable dented hexagon, there exists some $k$ independent of $a$ so that

$$
\mathrm{M}\left(H_{a, b, c, t,\left(u_{i}\right)_{1}^{m},\left(v_{j}\right)_{1}^{n}}\right)=k \cdot f_{b, c,\left(u_{i}\right)_{1}^{m},\left(v_{j}\right)_{1}^{n}}(a) .
$$

Then

$$
\begin{aligned}
\frac{\mathrm{M}\left(H_{a, b, c, t,\left(u_{i}\right)_{1}^{m},\left(v_{j}\right)_{1}^{n}}\right)}{\mathrm{M}\left(H_{\left.0, b, c, t,\left(u_{i}\right)_{1}^{m},\left(v_{j}\right)_{1}^{n}\right)}\right.} & =\frac{k \cdot f_{b, c,\left(u_{i}\right)_{1}^{m},\left(v_{j}\right)_{1}^{n}}(a)}{k \cdot f_{b, c,\left(u_{i}\right)_{1}^{m},\left(v_{j}\right)_{1}^{n}(0)}} \\
& =\frac{f_{b, c,\left(u_{i}\right)_{1}^{m},\left(v_{j}\right)_{1}^{n}(a)}}{f_{b, c,\left(u_{i}\right)_{1}^{m},\left(v_{j}\right)_{1}^{n}(0)}} \\
& =\frac{P(a, b+n, c+m)}{\prod_{i=1}^{m}\left(a+u_{i}\right)_{\underline{u_{i}}} \prod_{j=1}^{n}\left(a+v_{j}\right)_{\underline{v_{j}}}} \cdot \frac{\prod_{i=1}^{m}\left(0+u_{i}\right)_{\underline{u_{i}}} \prod_{j=1}^{n}\left(0+v_{j}\right)_{v_{j}}}{P(0, b+n, c+m)} \\
& =\frac{P(a, b+n, c+m)}{\prod_{i=1}^{m}\left(a+u_{i}\right)_{\underline{u_{i}}} \prod_{j=1}^{n}\left(a+v_{j}\right)_{\underline{v_{j}}}} \cdot \prod_{i=1}^{m}\left(u_{i}\right)_{\underline{u_{i}}} \prod_{j=1}^{n}\left(v_{j}\right)_{\underline{v}_{j}} .
\end{aligned}
$$

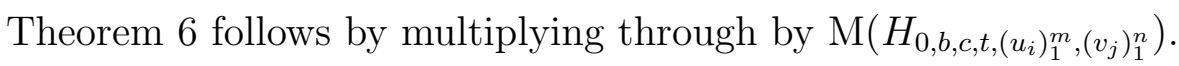

\section{Hexagons with Two Large Dents}

When the dents lying along each side of a dented hexagon are all next to each other, as in $H_{a, b, c, t,(u+i)_{i=1}^{m},(v+j)_{j=1}^{n}}$, the region has forced lozenges that form large triangular dents. Figure 10 depicts this forcing, and the region that results if the forced lozenges and dents are removed entirely.

The original goal of this paper was finding a general tiling function for hexagons with two large dents, and indeed Theorem 6 simplifies to a ratio of tilings of semiregular hexagons when the dents lying along each side are all adjacent: 


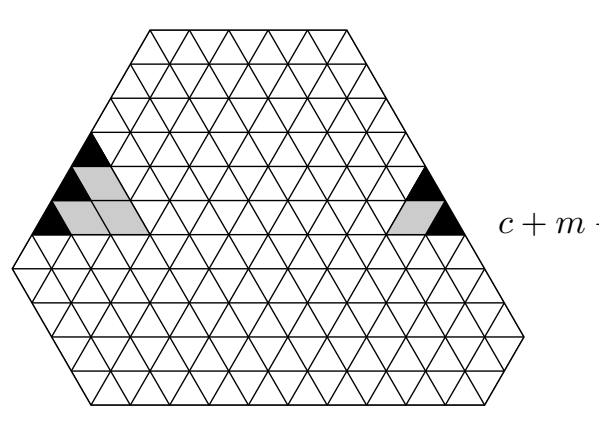

(a)

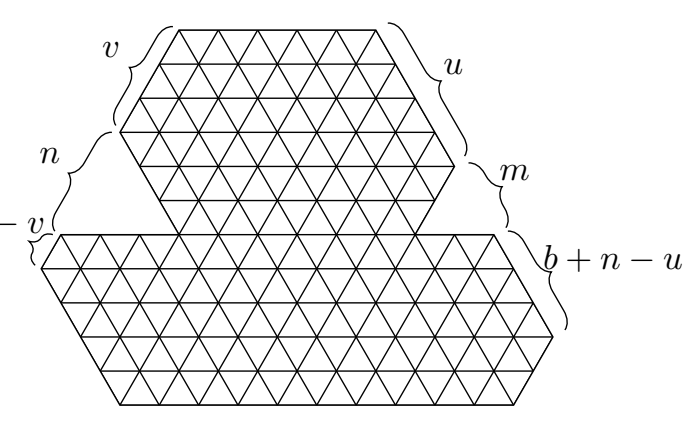

(b)

Figure 10: (a) $H_{5,4,2,5,(4+i)_{1}^{2},(3+j)_{1}^{3}}$; (b) the region with forced lozenges removed.

Corollary 11. A balanced dented hexagon $H_{a, b, c, t,(u+i)_{i=1}^{m},(v+j)_{j=1}^{n}}$ is tileable if and only if $u \geqslant n$ or $v \geqslant m$, in which case

$$
\begin{aligned}
\mathrm{M}\left(H_{a, b, c, t,(u+i)_{i=1}^{m},(v+j)_{j=1}^{n}}\right)= & \mathrm{M}\left(H_{0, b, c, t,(u+i)_{i=1}^{m},(v+j)_{j=1}^{n}}\right) \\
& \times \frac{P(a, b+n, c+m) P(u, b+n-u, m) P(v, c+m-v, n)}{P(a+u, b+n-u, m) P(a+v, c+m-v, n)} .
\end{aligned}
$$

This generalizes a specific case of a result by Lai who studied the problem when the southern borders of the dents are level (see Theorem 3.1 from [18], with $q=1$ ). We give an expression of that result below in the language of this paper; it follows from Corollary 11.

Corollary 12. Given a balanced dented hexagon $H_{a, b, c, m+n,(u+i)_{i=1}^{m},(v+j)_{j=1}^{n}}$ with $u+m=$ $v+n$, let $D:=u-n$. If $D<0$ the region has no tilings. Otherwise,

$$
\begin{aligned}
\mathrm{M}\left(H_{a, b, c, t,(u+i)_{i=1}^{m},(v+j)_{j=1}^{n}}\right)= & \frac{P(a, b+n, c+m) P(u, b-D, m) P(v, c-D, n)}{P(a+u, b-D, m) P(a+v, c-D, n)} \\
& \times \frac{P(c-D, n+m, b) P(D, n, m)}{P(c-D+n, m, D)} \cdot P(D, m, b-D) .
\end{aligned}
$$

Proof of Corollary 11. It is straightforward to check for arbitrary values that

$$
\begin{aligned}
& m>v, n>u \Longleftrightarrow \quad v<u+m \leqslant v+n, \text { and } \quad m+(u+m-v)>u+m \\
& \text { OR } \quad u<v+n \leqslant u+m, \text { and } \quad n+(v+n-u)>v+n .
\end{aligned}
$$

We shall show the second set of expressions hold if and only if the dented hexagon $H_{a, b, c, t,(u+i)_{1}^{m},(v+j)_{1}^{n}}$ has no tilings.

Suppose that $u+m \leqslant v+n$ (meaning the southern border of the eastern dent is weakly north of the southern border of the western dent) and the region has no tilings. Then $\left(\mu_{N}-N\right)$ is maximized at $N=u+m$ : so $\mu_{u+m}>u+m$. If $v>u+m$ then $\mu_{u+m}=m \leqslant u+m$, giving a contradiction. So it must be that $v \leqslant u+m$. This implies $\mu_{u+m}=m+(u+m-v)>u+m$, so that the first line on the right side of (20) holds. The 


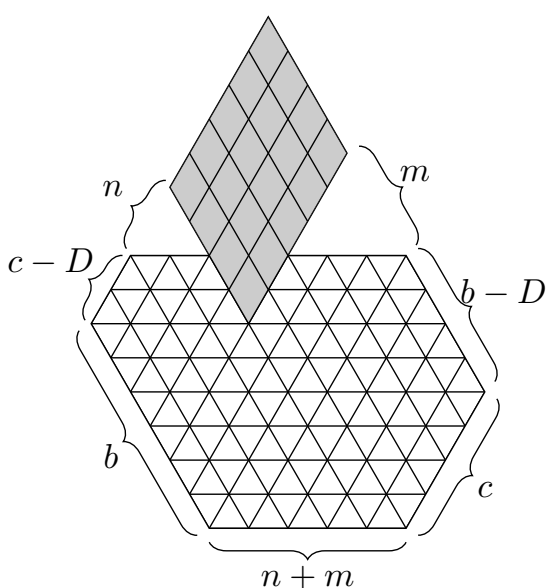

(a)

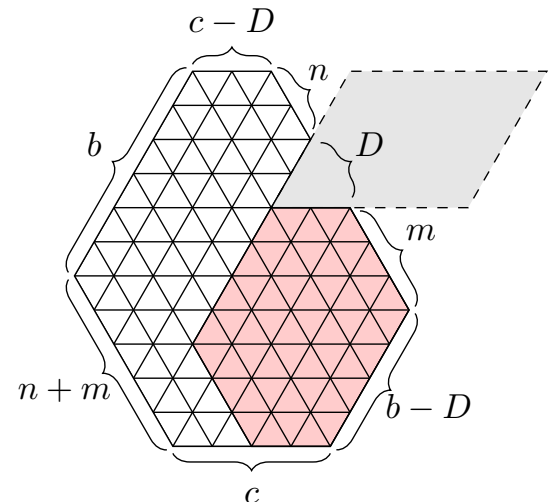

(b)

Figure 11: (a) $H_{0,6,4,5,(4+i)_{1}^{3},(5+j)_{1}^{2}}$ with forced lozenges; (b) the region interpreted as a hexagon with one dent $\left(H_{D, m, b-D}\right.$ shaded red).

argument works in reverse: if $v<u+m \leqslant v+n$ and $(u+m)-v>u$ then $\mu_{u+m}>u+m$; so these three inequalities are equivalent to the eastern dent being weakly north of the western dent and the region having no tilings.

The inequalities $u<v+n \leqslant u+m$ and $n+(v+n-u)>v+n$ are equivalent to the western dent being weakly north of the eastern dent and the region having no tilings.

The formula given follows immediately from Theorem 6 .

Proof of Corollary 12. The region $H_{0, b, c, m+n,(u+i)_{i=1}^{m},(v+j)_{j=1}^{n}}$ has forced lozenges that when removed give a region congruent to $H_{c-D, n+m, b-D, D,(n+i)_{i=1}^{D}, \vec{\varnothing}}$, as depicted in figure 11.

The region $H_{0, n+m, b-D, D,(n+i)_{i=1}^{D}, \vec{\varnothing}}$ also has forced lozenges, that when removed give a semiregular hexagon $H_{D, m, b-D}$. The result then follows by applying Corollary 11 to both $H_{a, b, c, t,(u+i)_{i=1}^{m},(v+j)_{j=1}^{n}}$ and $H_{c-D, n+m, b-D, D,(n+i)_{i=1}^{D}, \vec{\varnothing}}$.

\section{$7 \quad$ Final Remarks}

The method of proof used for Corollary 12 is applicable whenever the tiling function of $H_{0, b, c, t, \vec{u}, \vec{v}}$ is simple to express. For example, let $H=H_{0, b, c, t,(u+i)_{1}^{m},(v+j)_{1}^{n}}$ be a region with $v_{n}=1$, as depicted in Figure 12 (a).

Observe that the blue split-line in the figure partitions the region into two unbalanced hexagons $H_{n, b, 0,1}$ and $H_{m-1, b+n-u, c-1,1}$. It can be seen by modifying the proof of the Region Splitting Lemma that if $R$ is a balanced region with a partition into regions $P, Q$, so that triangles in $P$ which are adjacent to triangles in $Q$ are all of the same orientation, and this orientation is in excess within $P$ by some amount, say $d$, then all tilings of $R$ include exactly $d$ lozenges covering one triangle from $P$ and one triangle from $Q$.

It follows that all tilings of the entire region must include exactly one lozenge which 


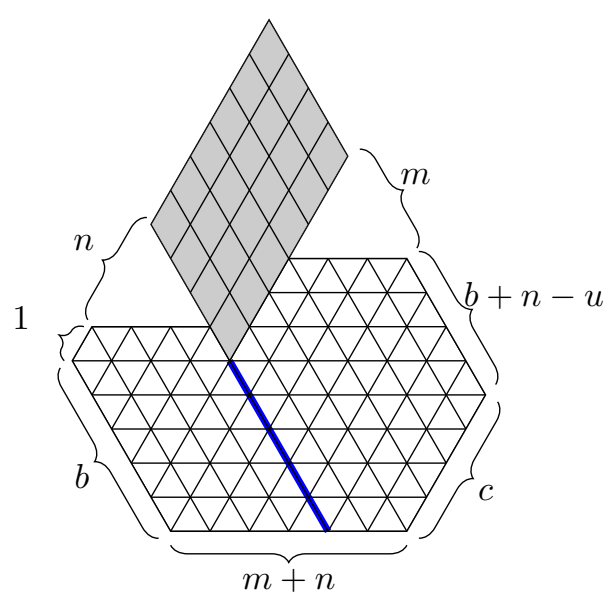

(a)

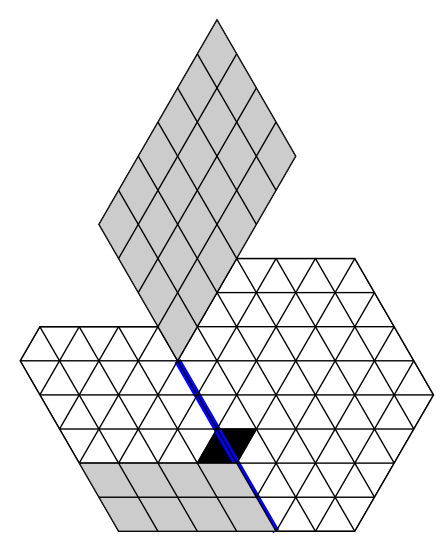

(b)

Figure 12: (a) $H_{0, b, c, \vec{u}, \vec{v}}$ with $\underline{v_{n}}=1$ and a split-line; (b) $R_{3}$ with forced lozenges shaded grey.

crosses the split-line. Let $S_{i}$ be the set of tilings of the region so that the split-line-crossing lozenge's northern border is $i$ units north of $H$ 's southern side. Let $R_{i}$ be the region obtained by removing that lozenge from $H$ and observe that $S_{i}$ has a natural bijection with the tilings of $R_{i}$. Furthermore, Lemma 1 applies to each region $R_{i}$ with respect to the blue split-line, and partitions $R_{i}$ into two regions with known tiling functions:

$$
\begin{aligned}
\mathrm{M}\left(R_{i}\right)= & \mathrm{M}\left(H_{1, n, b+1-i}\right) \mathrm{M}\left(H_{m-1, b+n-u, c-1,1,(i), \vec{\varnothing})}\right. \\
= & \frac{P(m-1, b+n-u, c)(b+n-u) !}{n !(c-1) !(b+m+n-u-1) !} \\
& \times(b+2-i)_{n}(b+n+2-u-i)_{c-1}(i)_{m-1} \\
\mathrm{M}\left(H_{\left.a, b, c, t,(u+i)_{1}^{m},(v+j)_{1}^{n}\right)=}\right. & \frac{P(a, b+n, c+m) P(u, b+n-u, m)(v+1)_{n}}{P(a+u, b+n-u, m)(a+v+1)_{n}} \times \sum_{i=1}^{b+n+1-u} \mathrm{M}\left(R_{i}\right)
\end{aligned}
$$

A similar calculation could be made when $1 \leqslant \underline{v_{n}} \leqslant m$, indexing over the positions of $\left|\underline{v_{n}}\right|$ distinct lozenges which cross the split-line.

We can apply this method to a different family of dented hexagons, with $v$ arbitrary, $u<b+1$, and $n=1$, employing a split-line which cuts southwest from the eastern dent, as depicted in Figure 13.

Again, each tiling of this region has exactly one lozenge which crosses the split-line. If $R_{i}$ is obtained by removing the lozenge in the $i$ th position from the southwest end of the split-line, then tilings of $H_{0, b, c, m+1,(u+i)_{1}^{m},(v+1)}$ are in bijection with the union of the tilings of $\left\{R_{i}\right\}_{i=1}^{c+m-v+1}$ :

$$
\mathrm{M}\left(R_{i}\right)=\mathrm{M}\left(H_{1, u-1, c+m+1-v-i}\right) \mathrm{M}\left(H_{b-u, c, m, 1,(i), \vec{\varnothing}}\right)
$$




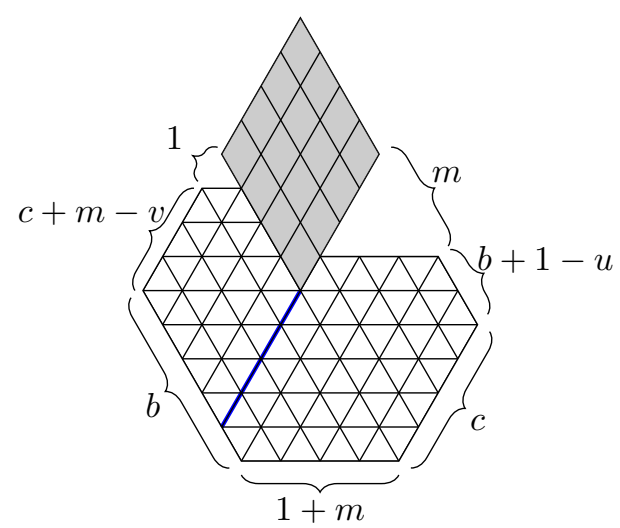

(a)

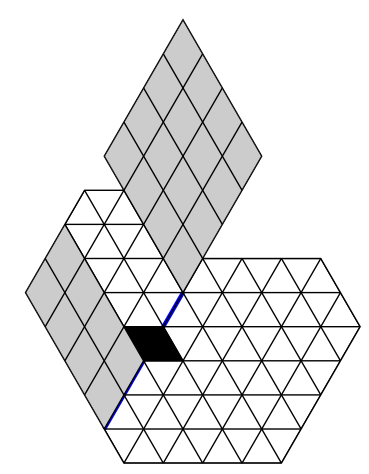

(b)

Figure 13: (a) $H_{0, b, c, m+1, \vec{u}, \vec{v}}$ with $n=1$ and a split-line; (b) $R_{3}$ with forced lozenges shaded grey.

$$
\begin{aligned}
& =\frac{P(b-u, c, m+1) c !}{(u-1) !(b-u+c) ! m !} \times(c+m-v-i+2)_{u-1}(c-i+2)_{m}(i)_{b-u} \\
H_{a, b, c, m+1,(u+i)_{1}^{m},(v+1)}= & \frac{P(a, b+1, c+m) P(u, b+1-u, m)(v+1)_{a}}{P(a+u, b+1-u, m)(c+m+1)_{a}} \times \sum_{i=1}^{c+m-v+1} \mathrm{M}\left(R_{i}\right) .
\end{aligned}
$$

We note some dead ends. We have shown that the tiling function for the dented hexagon $H_{a, b, c, t, \vec{u}, \vec{v}}$ may be given as a polynomial of entirely linear factors (of $a$ when the other parameters are fixed). The same is not true for obvious other parameters of the region, such as $c$ or $b$. Similarly when dents lie along more than two sides of the hexagon, or along short sides of the hexagon, the tiling function of the region could not be interpreted as a polynomial of linear factors over any obvious single parameter of the region.

\section{Acknowledgments.}

The author would like to thank M. Ciucu for introducing me to this subject, for his insights into this problem, and for his feedback on this paper.

\section{References}

[1] S. H. Byun, A short proof of two shuffling theorems for tilings and a weighted generalization (2020), submitted, available at arXiv:1906.04533 [math.CO].

[2] M. Ciucu and I. Fischer, Lozenge tilings of hexagons with arbitrary dents, Adv. Appl. Math. 73 C (2016), 1-22.

[3] M. Ciucu, Enumeration of perfect matchings in graphs with reflective symmetry, J. Combin. Theory Ser. A 77 (1997), no. 1, 67-97. 
[4] M. Ciucu, T. Eisenkölbl, C. Krattenthaler, and D. Zare, Enumeration of lozenge tilings of hexagons with a central triangular hole, J. Combin. Theory Ser. A 95 (2001), 251-334.

[5] M. Ciucu, T. Lai, and R. Rohatgi, Tilings of hexagons with a removed triad of bowties (2020), available at arXiv:1909.04070 [math.CO].

[6] M. Ciucu and T. Lai, Lozenge tilings of doubly-intruded hexagons, J. Combin. Theory Ser. A 167 (2019), 294-339.

[7] H. Cohn, M. Larsen, and J. Propp, The shape of a typical boxed plane partition, New York J. Math. 4 (1998), 137-165.

[8] G. David and C. Tomei, The problem of the calissons, Amer. Math. Monthly 96 (935) (1989), 429-431.

[9] T. Eisenkölbl, Rhombus tilings of a hexagon with three fixed border tiles, J. Combin. Theory Ser. A 88 (1999), 368-378.

[10] M. Gelfand and M. L. Tsetlin, Finite-dimensional representations of the group of unimodular matrices, Dokl. Akad. Nauk. 71 (1950), 825-828 (Russian); English transl., Izrail M. Gelfand: Collected Papers 2 (1988), 653-656.

[11] I. Gessel and X. Viennot, Binomial determinants, paths, and hook length formulae., Adv. in Math. 58 (1985), no. 3, 300-321.

[12] _ Determinants, paths, and plane partitions (1989), preprint.

[13] T. Gilmore, Inverting the Kasteleyn matrix for holey hexagons (2017), submitted, available at arXiv: 1701.07092 [math. C0].

[14] S. Karlin and J. McGregor, Coincidence probabilities, Pacific J. Math. 9 (1959), 11411164.

[15] P. W. Kasteleyn, Graph theory and crystal physics., Graph Theory and Theoretical Physics (1967), 43-110.

[16] E. Kuo, Applications of graphical condensation for enumerating matchings and tilings, Theoret. Comput. Sci. 319 (2004), no. 1-3, 29-57.

[17] T. Lai, Enumeration of hybrid domino-lozenge tilings, J. Combin. Theory Ser. A 122 (2014), 53-81.

[18] _ A q-enumeration of lozenge tilings of a hexagon with three dents, Adv. in Appl. Math. 82 (2017), 23-57.

[19] _ A shuffling theorem for reflectively symmetric tilings (2019), submitted, available at arXiv: 1905.09268 [math.CO].

[20] _ A shuffing theorem for centrally symmetric tilings (2019), submitted, available at arXiv:1906.03759 [math.CO].

[21] T. Lai and R. Rohatgi, A shuffling theorem for lozenge tilings of doubly-dented hexagons (2019), submitted, available at arXiv:1905.08311 [math.CO]. 
[22] B. Lindström, On the vector representations of induced matroids, Bull. Lond. Math. Soc. 5 (1973), 85-90.

[23] P. A. MacMahon, Combinatory Analysis, Vol. 2, Cambridge University Press, 1916; reprinted in P. A. MacMahon, Combinatory Analysis, Vol. 1-2, Chelsea, New York, 1960.

[24] H. N. V. Temperley and M. E. Fisher, Dimer problem in statistical mechanics - an exact result, Philos. Mag. (8) 6 (1961), 1061-1063. 